\title{
Eph/ephrin signalling serves a bidirectional role in lipopolysaccharide-induced intestinal injury
}

\author{
YING XIONG ${ }^{1}$, KAI-XUE LI ${ }^{2}$, HONG WEI ${ }^{2}$, LU JIAO $^{2}$, SHAO-YONG YU $^{3}$ and LI ZENG ${ }^{2}$ \\ ${ }^{1}$ Department of Gastroenterology, Shenzhen Longhua District Central Hospital, Shenzhen, Guangdong 518110;
${ }^{2}$ Department of Gastroenterology, The Second People's Hospital of Shenzhen, Shenzhen, Guangdong 518035, P.R. China;
${ }^{3}$ Division of Gastroenterology, Johns Hopkins University School of Medicine, Baltimore, MD 21205-2195, USA
}

Received December 27, 2017; Accepted June 6, 2018

DOI: $10.3892 / \mathrm{mmr} .2018 .9169$

\begin{abstract}
A growing body of evidence has demonstrated that Eph/ephrin signalling may serve a central role in intestinal diseases. However, whether erythropoietin-producing hepatocellular (Eph)/ephrin signalling is associated with the development of post-infectious irritable bowel syndrome (PI-IBS) is still unknown. In the present study, the role of Eph/Ephrin signalling in lipopolysaccharide (LPS)-induced intestinal injury was evaluated in vivo and in vitro. LPS treatment significantly increased the levels of proinflammatory mediators [monocyte chemoattractant protein-1, tumour necrosis factor $\alpha$, interleukin (IL)-1 $\beta$, IL-6, intercellular adhesion molecule 1 and vascular cell adhesion molecule-1], activated the EphA2-Ephrin A1, protein kinase B (Akt)-nuclear factor $(\mathrm{NF})-\kappa \mathrm{B}, \mathrm{Src}-\mathrm{NF}-\kappa \mathrm{B}$ and $\mathrm{Wnt} / \beta$-catenin signalling pathways, and inhibited EphB1-Ephrin B3 signalling in colon tissues, and primary cultured enteric neuronal and glial cells. Notably, EphA2 monoclonal antibody (mAb) treatment or Ephrin B3 overexpression could partially alleviate the LPS-induced upregulation of proinflammatory mediators, and Akt-NF- $\kappa \mathrm{B}$, Src-NF- $\kappa \mathrm{B}$ and $\mathrm{Wnt} / \beta$-catenin signalling pathways. In addition, EphA2 mAb treatment could partially inhibit LPS-induced inactivation of EphB-Ephrin B3 signalling, while Ephrin B3 overexpression could abrogate LPS-induced activation of EphA2-Ephrin A1 signalling. EphB1/Ephrin B3 signalling may antagonise the EphA2/Ephrin A1-dependent pathway following LPS treatment. The results associated with the EphA2 signaling pathway, indicated that Eph/ephrin signalling may serve a bidirectional role in LPS-induced intestinal
\end{abstract}

Correspondence to: Professor $\mathrm{Li}$ Zeng, Department of Gastroenterology, The Second People's Hospital of Shenzhen, 3002 Sungang West Road, Futian, Shenzhen, Guangdong 518035, P.R. China

E-mail: gastroenterology@163.com

Key words: post-infectious irritable bowel syndrome, lipopolysaccharide, erythropoietin-producing hepatocellular receptor, ephrin injury. Eph/ephrin signalling may be a novel therapeutic target for LPS-induced intestinal injury and potentially PI-IBS.

\section{Introduction}

Irritable bowel syndrome (IBS) is one of the most prevalent functional gastrointestinal disorders (1). It is characterised by the presence of recurrent or chronic abdominal pain or discomfort and bloating (2); 3 and 35\% patients with IBS develop post-infectious IBS (PI-IBS) after acute gastrointestinal infection (3). Although it is not a lethal disorder, PI-IBS jeopardises the quality of life and remains a substantial burden on the health care system. The pathological mechanism of PI-IBS remains obscure, although growing evidence has supported that abnormal motility, low-grade inflammation, dysfunction of enteric nervous system (ENS), and visceral hypersensitivity are associated with the development of PI-IBS (4-6). Of these, dysfunction of the ENS is considered an important factor causing inflammation and increased intestinal permeability (7). ENS is regarded as a separate and the largest component of the peripheral nervous system (8). The ENS plays a central role in gastrointestinal motility, the maintenance of the epithelial barrier and local immune response (9). Recent findings considered the presence of structural and functional changes in the ENS as the underlying pathophysiology in acute gastrointestinal-stress-related PI-IBS symptoms $(10,11)$. However, the mechanisms by which the ENS contributes to the development of PI-IBS are still incompletely understood.

The erythropoietin-producing hepatocellular (Eph) receptor family is evolutionarily conserved and the largest of the receptor tyrosine kinase families (12). Eph receptor family can be divided into A or B subgroups, based on their specific affinities for different subsets of ephrin ligands (13). Eph/ephrin signalling plays a central role in contact-dependent communication between cells, differentiation of the ENS, inflammatory response, epithelial barrier function and the restoration of the injured intestinal epithelium (14-16).

In the present study, we aimed to characterise the role of Eph/ephrin signalling in lipopolysaccharide (LPS)-induced intestinal injury in vivo and in vitro. Our results showed that Eph/ephrin signalling play a bidirectional role in LPS-induced intestinal injury in vivo and in vitro. Eph/ephrin signalling 
might be a novel therapeutic target for LPS-induced intestinal injury and even PI-IBS.

\section{Materials and methods}

Reagents. Escherichia coli LPS (E. coli 0127: B8), collagenase $\mathrm{XI}$, dispaseI, sorbitol, insulin and EGF were purchased from Sigma-Aldrich (Sigma-Aldrich; Merck KGaA, Darmstadt, Germany); EphA2 monoclonal antibody (mAb) and mouse IgG was purchased from Abcam (Cambridge, MA, USA); Foetal bovine serum (FBS), Dulbecco's modified Eagle's medium (DMEM), L-glutamine, penicillin and streptomycin were purchased from Gibco (Thermo Fisher Scientific, Inc., Waltham, MA, USA). ELISA kits were purchased from Thermo Fisher Scientific, Inc.

LPS-induced intestinal injury in vivo. Male BALB/c mice (20-24 g) were purchased from Beijing Vital River Laboratory Animal Technology Co., Ltd. (Beijing, China). All animal procedures were performed in accordance with the National Institutes of Health Guidelines on the Use and Care of Animals, with approval from the Institutional Animal Experiment Committee of the Second People's Hospital of Shenzhen. All efforts have been made to minimize animal suffering and the number of animals used. They were housed in animal care facilities under controlled conditions of temperature $\left(23 \pm 1^{\circ} \mathrm{C}\right)$, humidity $(50 \pm 10 \%)$, with an alternating $12 \mathrm{~h} \mathrm{light/dark} \mathrm{cycle} \mathrm{and} \mathrm{free} \mathrm{access} \mathrm{to} \mathrm{food} \mathrm{and}$ water. After 7 days of environmental adaption, 30 mice were randomly divided into three groups: i) PBS+IgG treatment group; ii) LPS+IgG treatment group; and iii) LPS+EphA2 $\mathrm{mAb}$ treatment group. LPS $(100 \mu \mathrm{g} / \mathrm{kg})$ was administered intravenously. $4 \mu \mathrm{g}$ of either mouse IgG or EphA2 mAb were administered intravenously 6 and $12 \mathrm{~h}$ after LPS treatment. The control group was given $4 \mu \mathrm{g}$ of mouse IgG 6 and $12 \mathrm{~h}$ after PBS treatment. At the end of treatment, the mice were anesthetized with a mixture of ketamine $(100 \mathrm{mg} / \mathrm{kg}$ body weight) and xylazine (10 mg/kg body weight). Mice ( $\mathrm{n}=10$ per group) were perfused transcardially with $0.9 \%$ saline ( $\mathrm{pH}$ 7.4), and the colon tissues were removed rapidly and carefully for ELISA and western blotting.

Cell culture and drug treatment. Small intestines were aseptically removed from 1-day-old BALB/c mice. The intestines were incubated in collagenase XI $(750 \mu \mathrm{g} / \mathrm{ml})$ and dispaseI $\left(250 \mu \mathrm{g} / \mathrm{ml}\right.$ ) at $37^{\circ} \mathrm{C}$ for $30 \mathrm{~min}$. Then the tissues were purified through an osmotic gradient with $2 \%$ sorbitol. The cell pellet was cultured in DMEM supplemented with $10 \%$ FBS, insulin $(0.25 \mathrm{U} / \mathrm{ml})$, EGF $(20 \mathrm{ng} / \mathrm{ml})$ and $1 \%$ penicillin $(100 \mathrm{U} / \mathrm{ml})$ /streptomycin $(100 \mathrm{mg} / \mathrm{ml})$ at $37^{\circ} \mathrm{C}$ in $5 \% \mathrm{CO}_{2}$ and $95 \%$ atmosphere. The purification of primary cultured enteric neuronal and glial cells was confirmed by immunofluorescence using mouse monoclonal to PGP9.5 (ab8189; Abcam) and rabbit polyclonal to GFAP (ab7260; Abcam). The primary cultured enteric neuronal and glial cells on culture day 7 were used. The primary cultured enteric neuronal and glial cells were incubated with LPS (50 ng/ml) for $24 \mathrm{~h}$. Cell viability was evaluated with the Cell Counting Kit-8 (CCK-8). The release of lactate dehydrogenase (LDH) was detected using the assay kit (Nanjing Jiancheng Bioengineering Institute, China).
ELISA. The levels of monocyte chemoattractant protein-1 (MCP-1), tumour necrosis factor $\alpha$ (TNF- $\alpha)$, interleukin-1 $\beta$ (IL-1 $\beta$ ), IL-6, intercellular adhesion molecule 1 (ICAM-1) and vascular cell adhesion molecule-1 (VCAM-1) in the colon tissues and primary cultured enteric neuronal and glial cells were measured by ELISA kits following the manufacturer's instructions.

Western blot analysis. Immunoblotting was performed by technicians who were blinded to the experimental groups. The protein extracts were loaded into 8-12\% Bis-Tris gels in a Bio-Rad slab gel apparatus (MiniPROTEAN Tetra cell; Bio-Rad Laboratories, Inc., Hercules, CA, USA) and electrophoretically transferred to a nitrocellulose membrane. Blots were probed with the following antibodies: EphA2 (ab5386), Ephrin A1 (ab199697), Ephrin B3 (ab101699), EphB1 (ab129103), Cadherin (ab6528), $\beta$-catenin (ab16051), AKT (ab8805), AKT1 (phospho S473) (ab18206), nuclear factor (NF)- $\mathrm{B}$ p65 (ab16502), Src1 (ab5407), Src (phospho Y418) (ab4816), $\beta$-actin (ab8227) and Lamin B (ab194109) were obtained from Abcam and were used at a 1:1,000 dilution, followed by the appropriate secondary antibodies. Bands were visualised using the enhanced chemiluminescence method (Super Signal CL-HRP Substrate System; Pierce; Thermo Fisher Scientific, Inc.), scanned with a densitometer (Bio-Rad Laboratories, Inc.) and analysed quantitatively with commercial equipment (Multi-Analyst Macintosh Software for Image Analysis Systems; Bio-Rad Laboratories, Inc.). At least three independent experiments were carried out.

Stable transfection. To establish ENS cells overexpressing Ephrin B3, ENS cells were transfected with Ephrin B3 lentiviral activation particles (Santa Cruz Biotechnology, Inc., Dallas, TX, USA) in complete medium with Polybrene (5 $\mu \mathrm{g} / \mathrm{ml})$ after reaching $60 \%$ confluency and incubated overnight. Stable Ephrin B3 activated clones were selected using puromycin dihydrochloride $(5 \mu \mathrm{g} / \mathrm{ml})$. The protein expression of Ephrin B3 was examined by Western blotting.

Statistical analysis. All experiments were performed a minimum of three times. Statistical analysis was performed using SPSS 19.0 software (IBM Corp., Armonk, IL, USA). Data are presented as the mean \pm standard deviation. Independent experiments were pooled when the coefficient of variance could be assumed identical. One-way analysis of variance was used to assess significant differences for multiple groups, followed by the post hoc Bonferroni's test. $\mathrm{P}<0.05$ was considered to indicate a statistically significant difference.

\section{Results}

LPS treatment increased the expression of EphA2 and Ephrin A1 but decreased the expression of EphB1 and Ephrin B3 in colon. Western blot analysis showed that LPS treatment increased EphA2 and Ephrin A1 protein expression but decreased EphB1 and Ephrin B3 protein expression compared with PBS treatment in colon $(\mathrm{P}<0.05$; Fig. 1A and B). These results implied that EphA2-Ephrin A1 signalling and EphB1-Ephrin B3 signalling might play a central role in LPS-induced intestinal injury. 
A

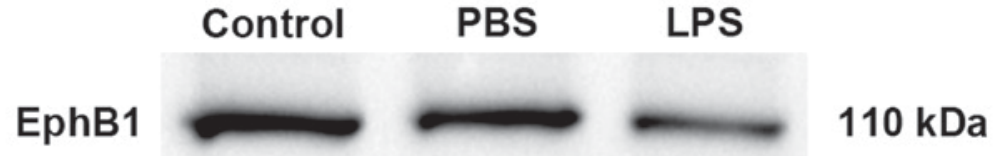

EphA2
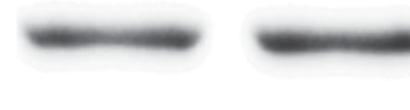

Ephrin B3

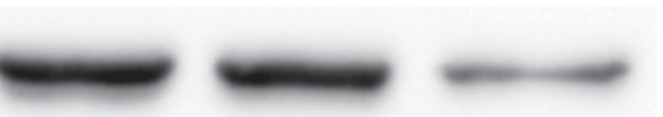

$108 \mathrm{kDa}$

\section{$45 \mathrm{kDa}$}

Ephrin A1
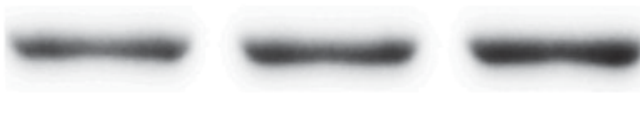

\section{$24 \mathrm{kDa}$}
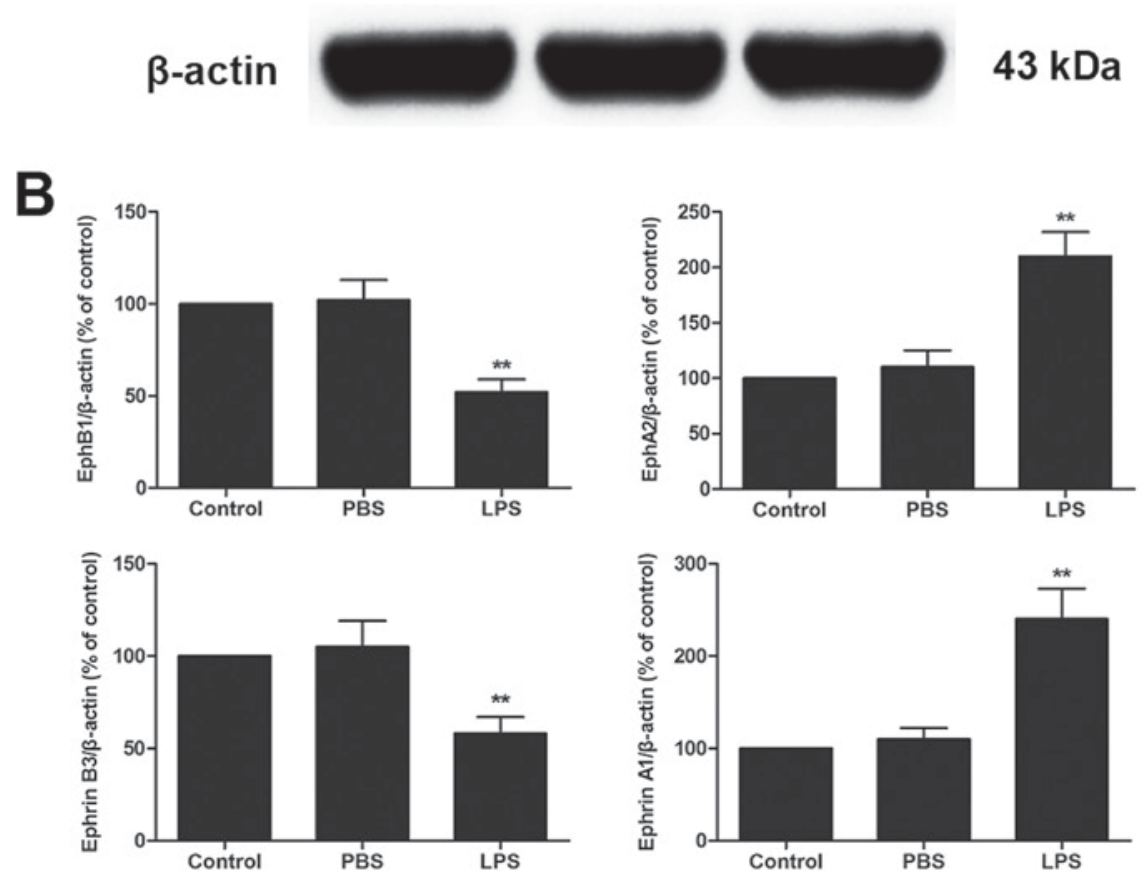

Figure 1. Effects of LPS on the expression of EphA2-Ephrin A1 and EphB1-Ephrin B3 signaling in vivo. (A) Western blot analysis of EphA2, Ephrin A1, Ephrin B3 and EphB1 expression in colon tissues. (B) Densitometry was performed to quantify the levels of the proteins. The results are expressed as the mean \pm standard deviation ( $\mathrm{n}=6 /$ group). ${ }^{* *} \mathrm{P}<0.01$ vs. vehicle (PBS) group. Eph, erythropoietin-producing hepatocellular; LPS, lipopolysaccharide.

EphA2 antagonism enhanced EphB1/Ephrin B3 signalling. After finding that LPS increased expression of EphA2 and Ephrin A1 in colon, we further investigated the effects of EphA2 mAb on EphA2-ephrin A1 signalling and EphB1-Ephrin B3 signalling. We confirmed that the increased expression of EphA2 and Ephrin A1 and the decreased expression of EphB1 and Ephrin B3 induced by LPS were partially inhibited by EphA2 mAb treatment ( $\mathrm{P}<0.05$; Fig. $2 \mathrm{~A}$ and $\mathrm{B})$. Therefore, the data implied that the interaction between EphA2-Ephrin A1 and EphB1-Ephrin B3 might be positively correlated with LPS-induced intestinal injury.

EphA2 antagonism attenuated LPS-enhanced expression of inflammatory cytokines. Since inflammatory cytokines are major mediators of LPS-induced intestinal injury, we detected the levels of inflammatory cytokines (MCP-1, TNF- $\alpha$, IL-1 $\beta$,
IL-6, ICAM-1 and VACM-1) in colon. Compared with mice in PBS+IgG group, the levels of inflammatory cytokines (MCP-1, TNF- $\alpha$, IL-1 $\beta$, IL-6, ICAM-1 and VACM-1) were significantly increased in colon of mice in $\mathrm{LPS}+\operatorname{IgG}$ group $(\mathrm{P}<0.05$; Fig. 3). Compared with mice with IgG treatment after LPS treatment, decreased expression of inflammatory cytokines including MCP-1 (254.31 \pm 32.96 vs. $677.25 \pm 71.11)$, TNF- $\alpha$ $(21.85 \pm 4.92$ vs. $45.08 \pm 6.12)$, IL-1 $\beta(2.43 \pm 0.36$ vs. $5.65 \pm 0.91)$,

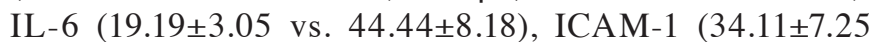
vs. $111.12 \pm 21.31)$, VACM-1 $(18.41 \pm 4.55$ vs. $34.19 \pm 5.09)$ were observed in the colon of mice with EphA2 mAb treatment $(\mathrm{P}<0.05$; Fig. 3).

EphA2 antagonism attenuated LPS-induced activation of Akt-NF- $\kappa B$ signalling, Src-NF- $\kappa B$ signalling and $W n t / \beta$-catenin signalling pathway. Since the protective effect 
EphB1

EphA2

Ephrin B3

Ephrin A1

$\beta$-actin

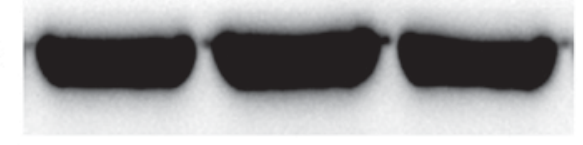

$110 \mathrm{kDa}$

$108 \mathrm{kDa}$

$45 \mathrm{kDa}$

$24 \mathrm{kDa}$

$43 \mathrm{kDa}$
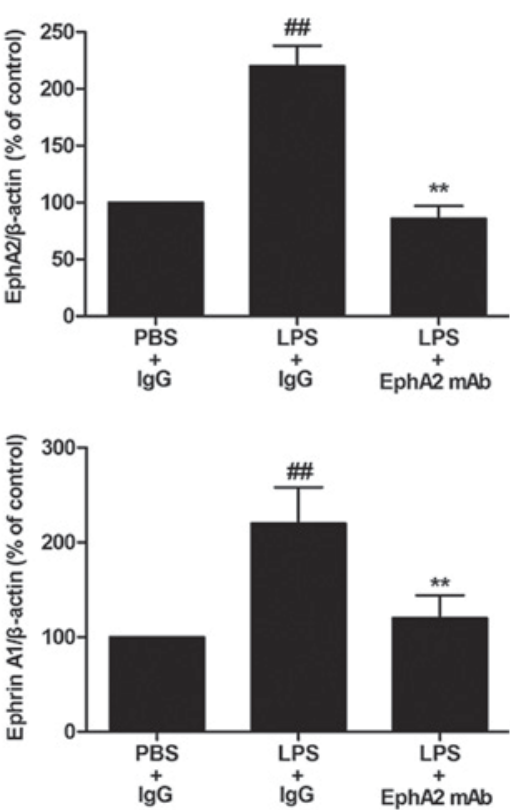

Figure 2. Effects of EphA2 antagonism on EphB1-Ephrin B3 expression in vivo. (A) Western blot analysis of EphA2, Ephrin A1, Ephrin B3 and EphB1 expression in the colon. (B) Densitometry was performed to quantify the levels of the proteins. The results are expressed as the mean \pm standard deviation ( $\mathrm{n}=6 /$ group). ${ }^{\# \#} \mathrm{P}<0.01$ vs. $\mathrm{PBS}+\mathrm{IgG}$ (control); ${ }^{* *} \mathrm{P}<0.01$ vs. LPS+IgG treatment. Eph, erythropoietin-producing hepatocellular; LPS, lipopolysaccharide; $\mathrm{mAb}$, monoclonal antibody; IgG, immunoglobulin G.

of EphA2 antagonism in LPS-induced intestinal injury, we wondered whether EphA 2 antagonism would affect Akt-NF- $\mathrm{B}$ signalling and Src-NF- $\kappa \mathrm{B}$ signalling pathways. The expression of Akt-NF- $\kappa$ B signalling and Src-NF- $\kappa$ B signalling were measured. As shown in Fig. 4A, compared with the PBS+IgG group, the LPS+IgG group showed a significant increase in Akt and Src phosphorylation in colon tissue $(\mathrm{P}<0.05)$. After EphA2 $\mathrm{mAb}$ treatment, the phosphorylation of Akt and $\mathrm{Src}$ induced by LPS was significantly reduced ( $\mathrm{P}<0.05$; Fig. 4A). Moreover, the phosphorylation of NF- $\kappa$ B p65 was increased significantly after LPS treatment $(\mathrm{P}<0.05)$. However, a significant decrease was observed in the phosphorylation of NF- $\mathrm{B}$ p65 after EphA2 mAb treatment, compared with the IgG treatment after LPS treatment $(\mathrm{P}<0.05$; Fig. 4A). This finding indicates that EphA2 signalling may be involved in LPS-induced acti-

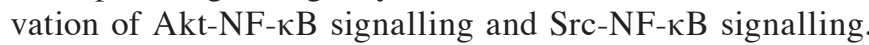

Activation of the Wnt/ $\beta$-catenin pathway plays a pivotal role in LPS-induced intestinal injury. To determine whether EphA2 antagonism could affect the Wnt/ $\beta$-catenin pathway, the expression level of $\mathrm{Wnt} / \beta$-catenin signalling proteins were determined by immunoblotting. As shown in Fig. 4B, compared with the PBS+IgG group, LPS could significantly increased nuclear translocation of $\beta$-catenin $(\mathrm{P}<0.05$; Fig. $4 \mathrm{~B})$. However, EphA 2 mAb treatment could significantly decreased nuclear translocation of $\beta$-catenin, compared with IgG treatment after LPS treatment $(\mathrm{P}<0.05$; Fig. $4 \mathrm{~B})$, suggesting that EphA2 signalling is responsible for LPS-mediated activation of the Wnt/ $\beta$-catenin pathway. Furthermore, LPS treatment could significantly reduce the expression of E-cadherin $(\mathrm{P}<0.05$; Fig. 4B), whereas treatment with EphA2 mAb significantly increased E-cadherin protein expression in colon $(\mathrm{P}<0.05$; Fig. 4B). These results demonstrate that EphA2 signaling may 

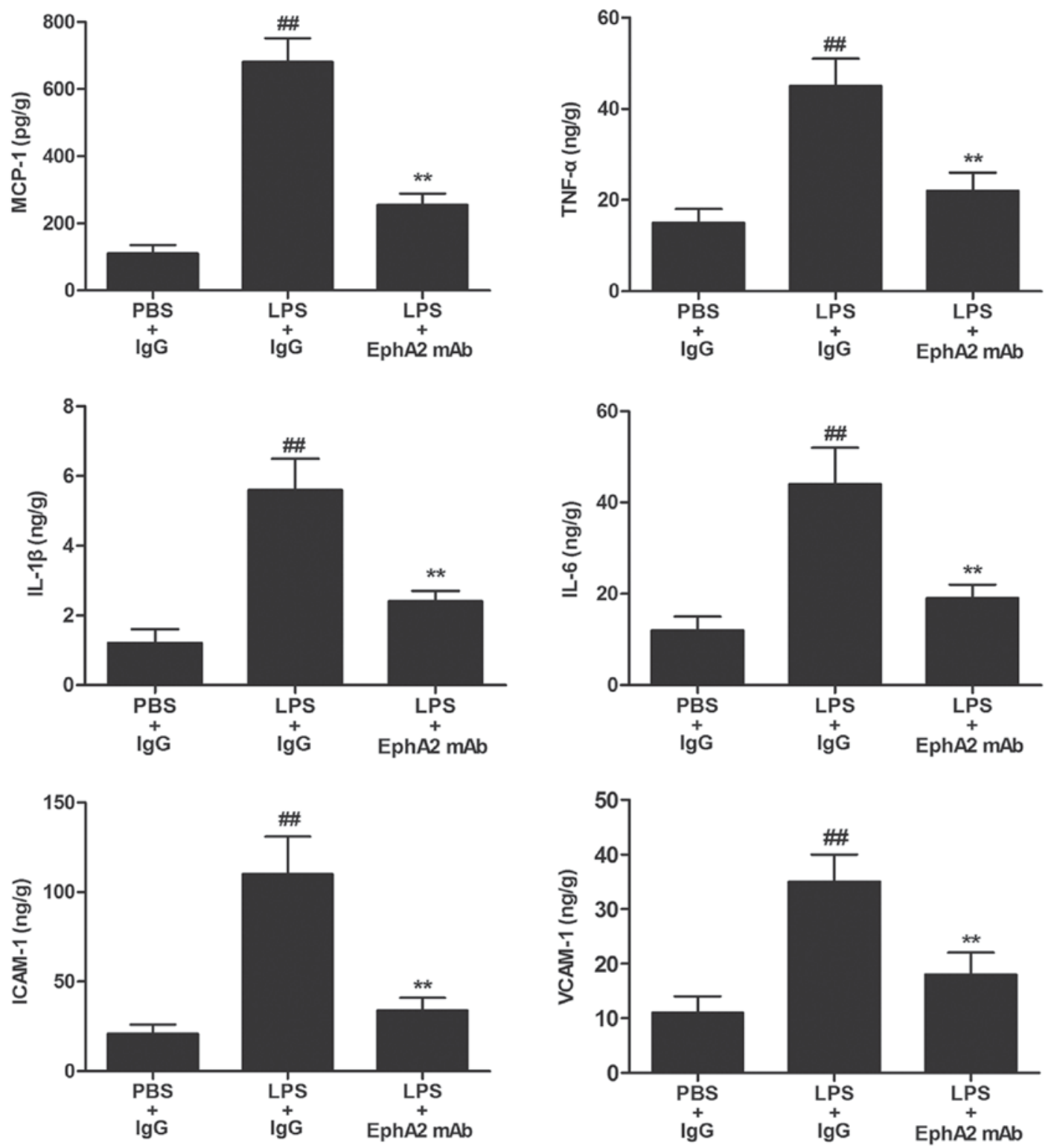

Figure 3. Effects of EphA2 antagonism on the levels of inflammatory cytokines in vivo. The levels of the inflammatory cytokines (MCP-1, TNF- $\alpha$, IL-1 $\beta$, IL-6, ICAM-1 and VCAM-1) in colon tissue lysates were measured by ELISA. The results are expressed as the mean \pm standard deviation ( $\mathrm{n}=6 / \mathrm{group}){ }^{\# \#} \mathrm{P}<0.01$ vs. $\mathrm{PBS}+\mathrm{IgG}$ (control); ${ }^{* *} \mathrm{P}<0.01$ vs. LPS+IgG treatment. Eph, erythropoietin-producing hepatocellular; LPS, lipopolysaccharide; IgG, immunoglobulin G; mAb, monoclonal antibody; MCP-1, monocyte chemoattractant protein-1; TNF- $\alpha$, tumour necrosis factor $\alpha$; IL, interleukin; ICAM-1, intercellular adhesion molecule 1; VCAM-1, vascular cell adhesion molecule-1.

regulate the expression of E-cadherin and adherens junction and epithelial hyperpermeability.

EphB1/Ephrin B3 signalling antagonised the EphA2/Ephrin Al-dependent pathway. The primary cultured enteric neuronal and glial cells were confirmed by immunofluorescence using mouse monoclonal to PGP9.5 and rabbit polyclonal to GFAP. The purification of primary cultured enteric neuronal and glial cells was more than $90 \%$ (data not shown). We further investigated the effects of Ephrin B3 overexpression on LPS-induced injury in primary cultured enteric neuronal and glial cells. A significant increase in the expression of EphA2 and Ephrin A1 and a significant decrease in EphB1 and Ephrin B3 were observed in LPS-treated primary cultured enteric neuronal and glial cells, compared with the control cells $(\mathrm{P}<0.05$; Fig. 5A). However, Ephrin B3 overexpression reversed LPS-induced up-regulation of EphA2 and Ephrin A1, and enhanced expression of EphB1 compared with the control group $(\mathrm{P}<0.05$; Fig. 5A). Importantly, Ephrin B3 overexpression significantly increased the cell viability and significantly decreased LDH leakage ( $\mathrm{P}<0.05$; Fig. 5B).

Ephrin B3 overexpression reversed LPS-enhanced expression of inflammatory cytokines in primary cultured enteric neuronal and glial cells. To examine whether Ephrin B3 overexpression negatively regulated LPS-induced inflammatory responses, we detected the levels of inflammatory cytokines (MCP-1, TNF- $\alpha$, IL-1 $\beta$, IL-6, ICAM-1 and VACM-1) in primary cultured enteric neuronal and glial cells. We found that the levels of inflammatory cytokines (MCP-1, TNF- $\alpha$, IL-1 $\beta$, IL-6, ICAM-1 and VACM-1) were significantly increased after LPS treatment, compared with the control 
A

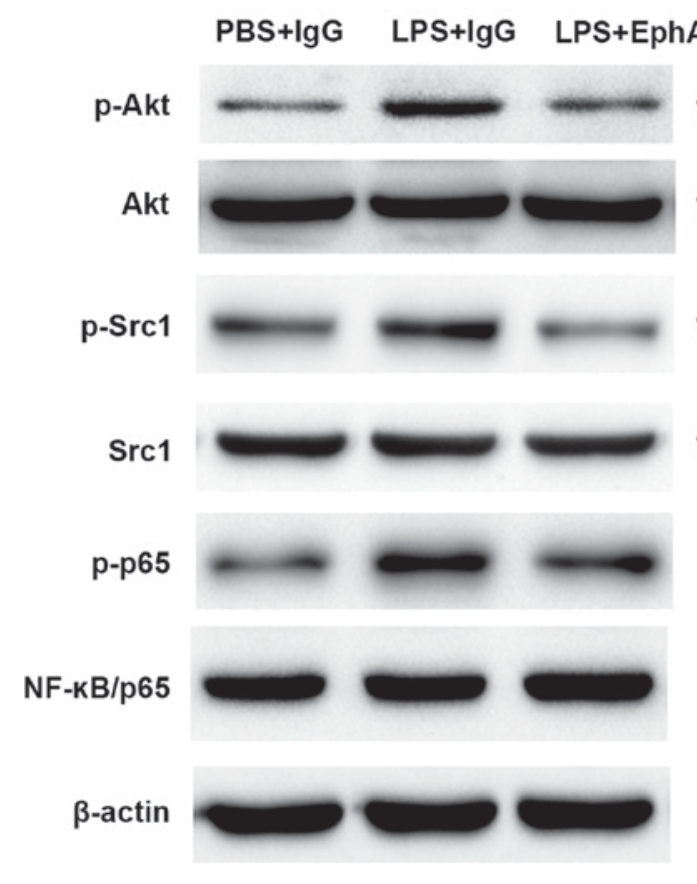

110 kDa

$110 \mathrm{kDa}$

$108 \mathrm{kDa}$

108 kDa

$64 \mathrm{kDa}$

64 kDa

$43 \mathrm{kDa}$
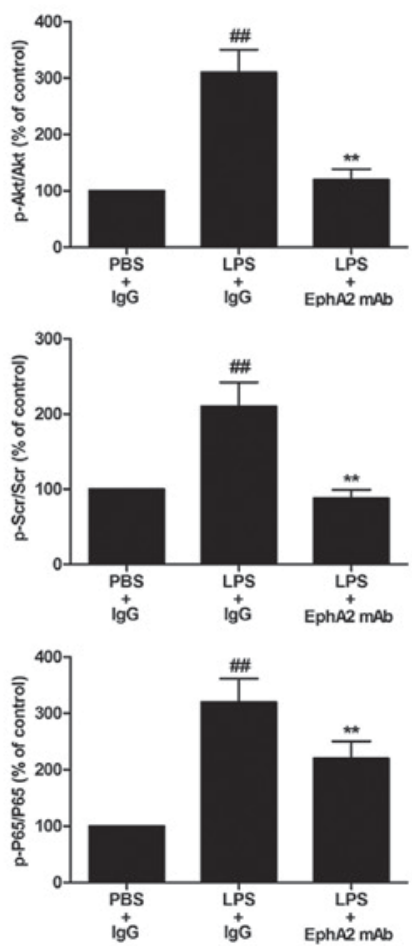

B

PBS+lgG LPS+lgG LPS+EphA2 mAb

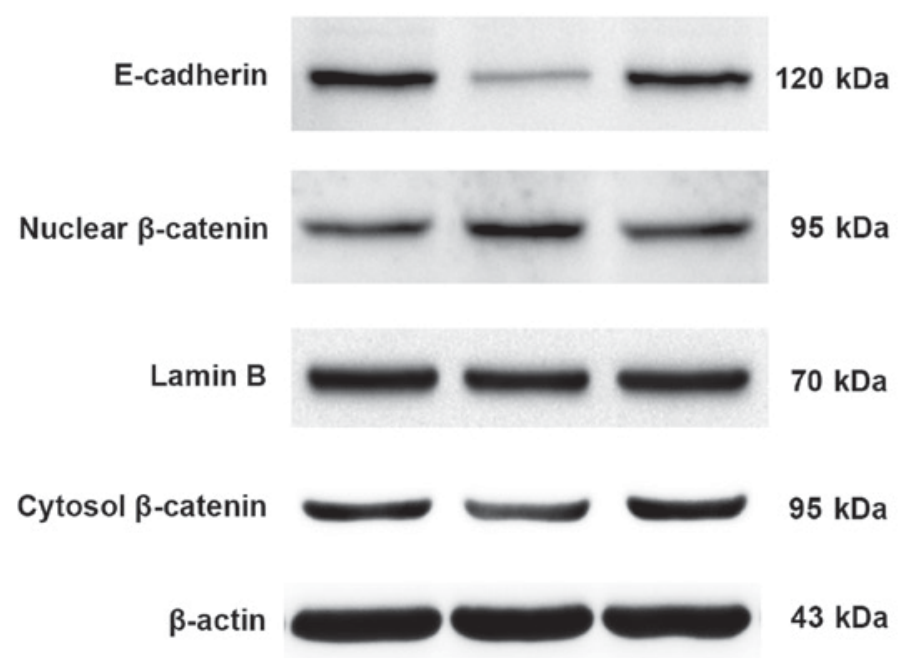

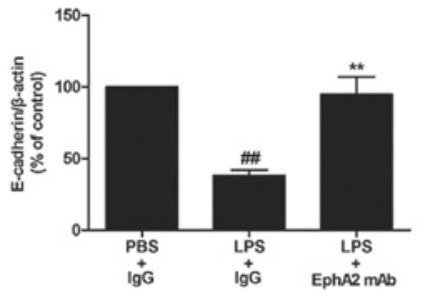
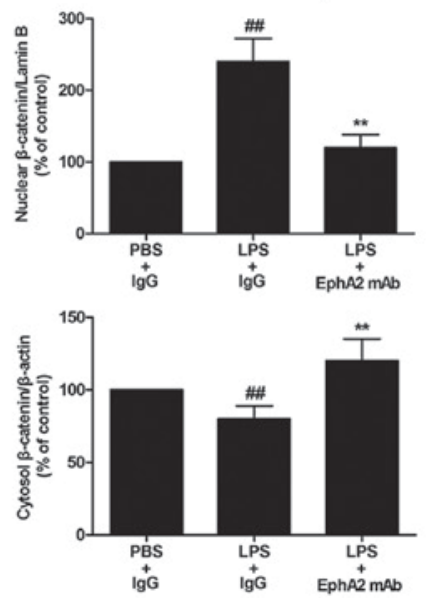

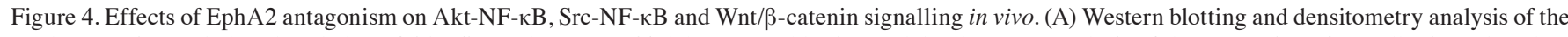
total expression and phosphorylation of Akt, Src and NF-кB p65. (B) Western blotting and densitometry analysis of the expression of E-cadherin and nuclear translocation of $\beta$-catenin. The results are expressed as the mean \pm standard deviation ( $\mathrm{n}=6 /$ group). ${ }^{\# \#} \mathrm{P}<0.01$ vs. $\mathrm{PBS}+\mathrm{IgG}$ (control); ${ }^{* *} \mathrm{P}<0.01 \mathrm{vs}$. LPS+IgG treatment. Eph, erythropoietin-producing hepatocellular; LPS, lipopolysaccharide; IgG, immunoglobulin G; Akt, protein kinase B; NF-кB, nuclear factor-кB; p-, phosphorylated; mAb, monoclonal antibody.

(P<0.05; Fig. 6). However, Ephrin B3 overexpression significantly reversed LPS-induced up-regulation of inflammatory cytokines $(\mathrm{P}<0.05$; Fig. 6$)$.

Ephrin B3 overexpression attenuated LPS-induced activa-

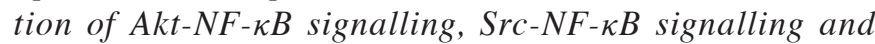
Wnt $/ \beta$-catenin signalling pathway in primary cultured enteric neuronal and glial cells. Because EphA2 signalling was shown to be crucial in LPS-induced activation of Akt-NF- $\kappa B$ signalling, Src-NF- $\kappa B$ signalling and $\mathrm{Wnt} / \beta$-catenin pathway, we examined the influence of Ephrin B3 overexpression in LPS-induced activation of Akt-NF- $\kappa B$ signalling, Src-NF- $\kappa B$ signalling and $\mathrm{Wnt} / \beta$-catenin pathway, thus exploring the potential link between bidirectional signalling mediated by Eph-Ephrins. Ephrin B3 overexpression significantly reversed the up-regulated phosphorylation of Akt, Src and NF-кB p65 

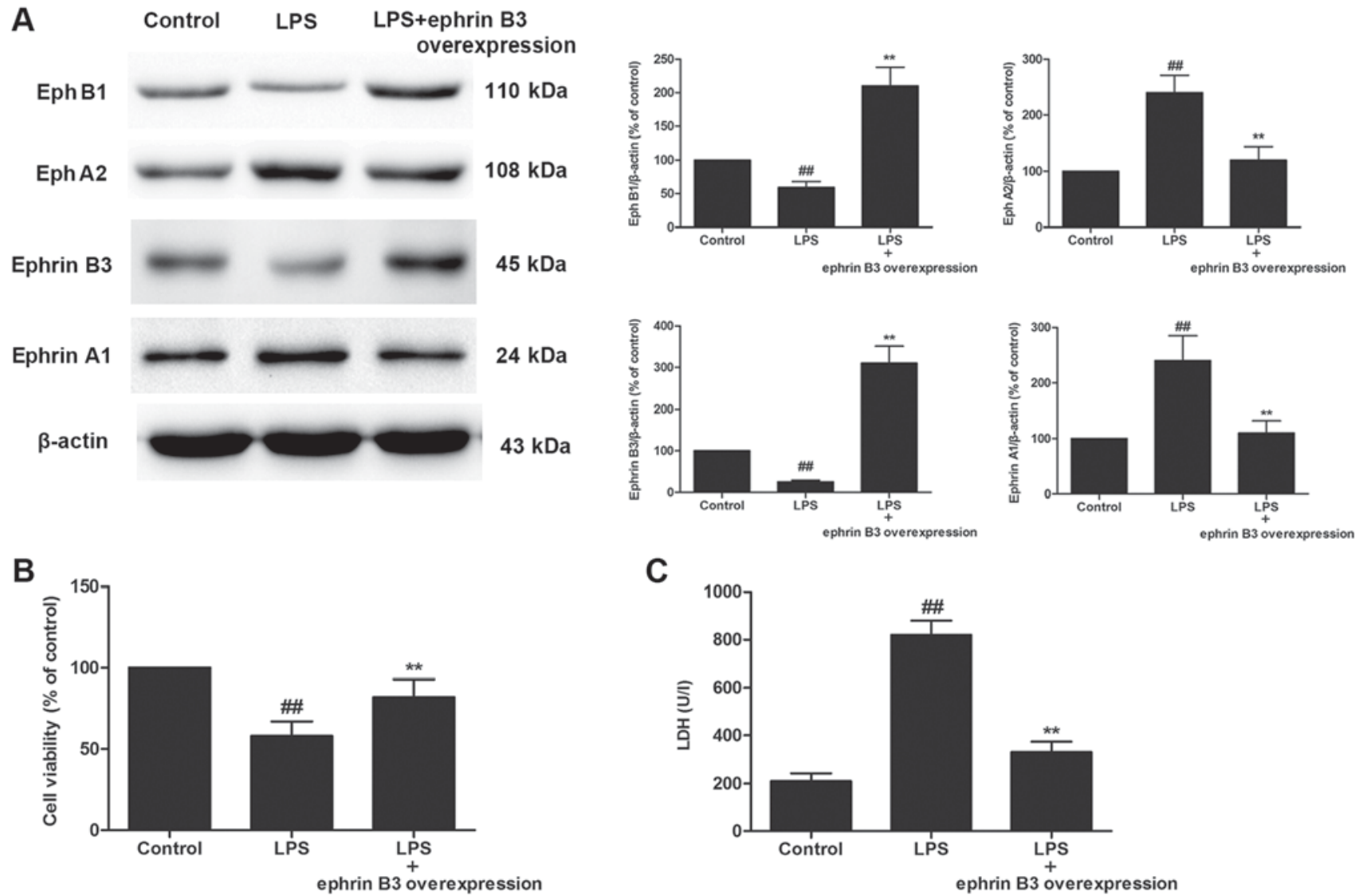

Figure 5. Effects of Ephrin B3 overexpression on EphA2-Ephrin A1 signaling in vitro. (A) Western blotting and densitometry analysis of EphA2, Ephrin A1, Ephrin B3 and EphB1 expression. (B) Effect of Ephrin B3 overexpression on LPS-induced injury in primary cultured enteric neuronal and glial cells using the Cell Counting Kit-8 assay. (C) Effect of Ephrin B3 overexpression on LPS-induced injury in primary cultured enteric neuronal and glial cells using the LDH leakage assay. The results are expressed as the mean \pm standard deviation $(n=3) .{ }^{\# \#} \mathrm{P}<0.01$ vs. control; ${ }^{* *} \mathrm{P}<0.01$ vs. LPS treatment. Eph, erythropoietin-producing hepatocellular; LPS, lipopolysaccharide; LDH, lactate dehydrogenase.

in LPS-treated primary cultured enteric neuronal and glial cells $(\mathrm{P}<0.05 ;$ Fig. 7A). In addition, Ephrin B3 overexpression significantly inhibited the nuclear translocation of $\beta$-catenin and increased expression of E-cadherin in LPS-treated primary cultured enteric neuronal and glial cells $(\mathrm{P}<0.05$; Fig. 7B).

\section{Discussion}

In recent years, the role of ENS in PI-IBS has gained increasing attention; however, there are still only a few studies addressing how the ENS contributes to PI-IBS. The data from our present work indicated that ENS responded to LPS-induced injury by activating proinflammatory mediators, and integrating the Eph-ephrin signalling-dependent downstream pathways (including the Akt-NF- $\mathrm{B}, \mathrm{Src}-\mathrm{NF}-\kappa \mathrm{B}$ and $\mathrm{Wnt} / \beta$-catenin pathways), which facilitated the development of PI-IBS. Importantly, we hypothesised that Eph receptors and ephrins activated bidirectional signalling networks which played a crucial role in PI-IBS.

Our studies demonstrated that EphA2-Ephrin A1 signalling was activated and EphB1-Ephrin B3 signalling was inactivated in LPS-induced injury in vivo and in vitro. Both the expression of the EphA2 receptor and Ephrin A1 were markedly increased in LPS-treated colon and ENS, while the expression levels of EphB1 receptor and Ephrin B3 were decreased. In addition, blocking of EphA2 receptor by EphA2 mAb partially ameliorated LPS-triggered injury and inflammatory responses in a mouse model. Moreover, Ephrin B3 overexpression reversed LPS-induced damage in primary cultured enteric neuronal and glial cells. In our experiments, we demonstrated that the Eph/ephrins singnaling played an opposing role and exerted different effects in LPS-induced intestial injury. We found that EphA2-Ephrin A1 mediated 'forward' signalling might contribute to LPS-induced injury, while Ephrin B3 dependent 'reverse' signalling may have a role in generating the negative regulation function after LPS treatment.

Signal transduction activated by ephrin binding to Eph receptors is associated with their interaction with specific intracellular pathways (17-19). Herein, our results suggested that increased Ephrin A1 might induce ligand-dependent EphA2 signalling, which further induced phosphorylation of Akt and Src. The activation of Akt and Src would trigger nuclear translocation of NF- $\mathrm{KB}$, which would lead to enhanced proinflammatory cytokine production. The role of Akt and Src pathways in modulating NF- $\kappa \mathrm{B}$ activation has been demonstrated in numerous cell populations $(20,21)$. Activation of Akt is required for the efficient localisation of $\mathrm{p} 65$ to the promoter regions of a specific subset of the NF-кB-targeted genes (22). In addition, it has been demonstrated that Src tyrosine kinases mediate the activation of NF- $\kappa B$ in LPS-induced injury, and that selective Src tyrosine kinase inhibitors could prevent this damage (23). In the present study, antagonising the EphA2-Ephrin A1 pathway by EphA2 

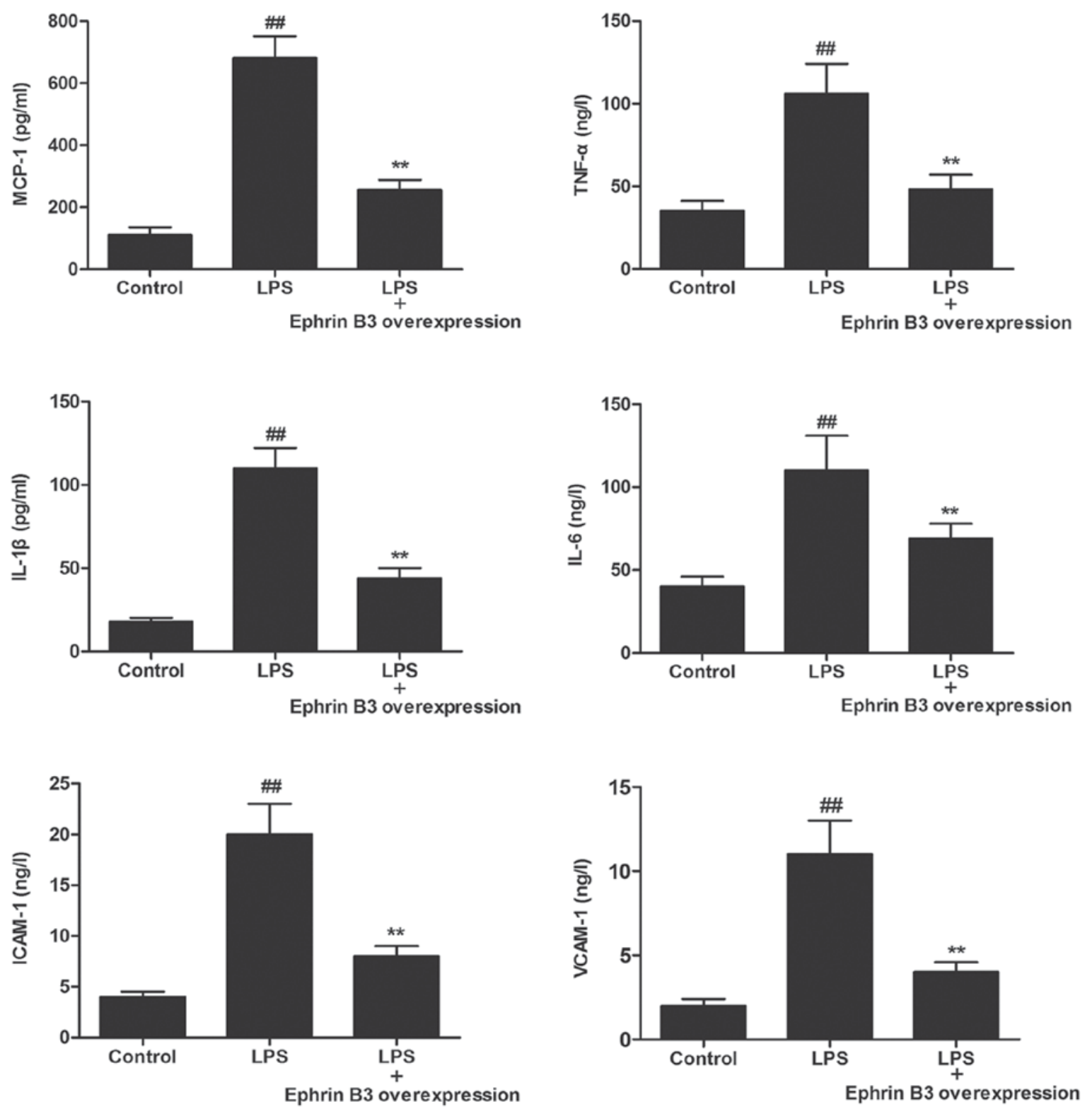

Figure 6. Effects of Ephrin B3 overexpression on inflammatory cytokines in vitro. The levels of inflammatory cytokines in primary cultured enteric neuronal and glial cells were determined by ELISA. The results are expressed as the mean \pm standard deviation $(n=3) .{ }^{\# \#} \mathrm{P}<0.01$ vs. control; ${ }^{* *} \mathrm{P}<0.01$ vs. LPS treatment. Eph, erythropoietin-producing hepatocellular; LPS, lipopolysaccharide; MCP-1, monocyte chemoattractant protein-1; TNF- $\alpha$, tumour necrosis factor $\alpha$; IL, interleukin; ICAM-1, intercellular adhesion molecule 1; VCAM-1, vascular cell adhesion molecule-1.

$\mathrm{mAb}$ treatment partially attenuated the phosphorylation of Akt-Src and NF- $\mathrm{BB}$, which suggested that LPS-induced activa-

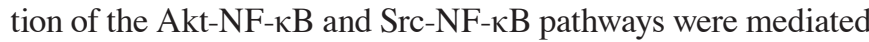
by EphA2-Ephrin A1 signalling.

Furthermore, our findings also indicated the involvement of EphA2 signalling in LPS-induced $N F-\kappa B$ activation via Wnt/ $\beta$-catenin as an upstream pathway. Enhanced EphA2 would facilitate the nuclear translocation of $\beta$-catenin protein, which is known to be a marker for hyperactivation of Wnt signalling (24). Many studies have demonstrated that constitutive activation of $\mathrm{Wnt} / \beta$-catenin signalling promoted the activation of NF-Kb $(25,26)$. Conversely, downregulation of the nuclear translocation of $\beta$-catenin and abrogation of Wnt signalling was exactly associated with inactivation of NF- $\kappa \mathrm{B}(27)$. Our study demonstrated that EphA2 mAb partially inhibited Wnt signalling by inhibiting the nuclear translocation of $\beta$-catenin in LPS-induced injury. This suggests that EphA2 might promote $\mathrm{NF}-\kappa \mathrm{B}$ activation via the $\mathrm{Wnt} / \beta$-catenin pathway.

Interestingly, our studies also demonstrated that EphB1-Ephrin B3 signalling might act to antagonise the EphA2-dependent pathway after LPS treatment. We found Ephrin B3 overexpression could reverse LPS-triggered injury, increased concentrations of cytokines, and activation of Akt-Src and NF- $\mathrm{B}$, which implied that enhanced Ephrin B3-dependent 'reverse' signalling served as a potentially negative regulator to counteract the EphA2-Ephrin A1 pathway. Previous studies have provided evidence that binding of EphB1 to Ephrin B3 led to a reduction and inactivation of Src in striatal neurons (28). In contrast, in cortical interneurons binding of EphB1 to Ephrin B3 would enhance the phosphorylation of Src (29). Consistent with our data, it has been demonstrated that the activation of EphrinB-dependent 'reverse' signalling could downregulate 
A

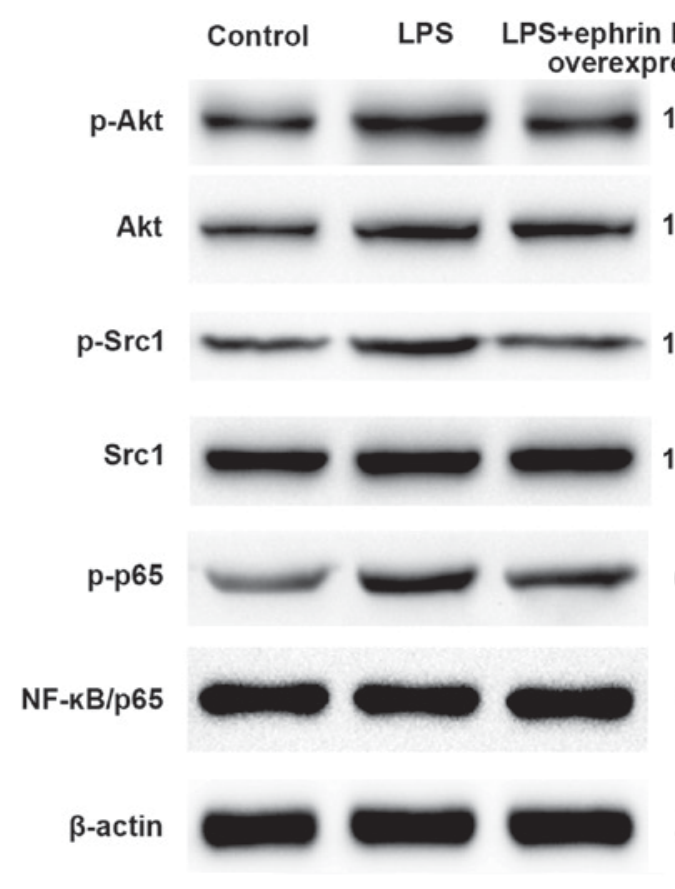

$108 \mathrm{kDa}$

108 kDa

$64 \mathrm{kDa}$

$64 \mathrm{kDa}$

$43 \mathrm{kDa}$
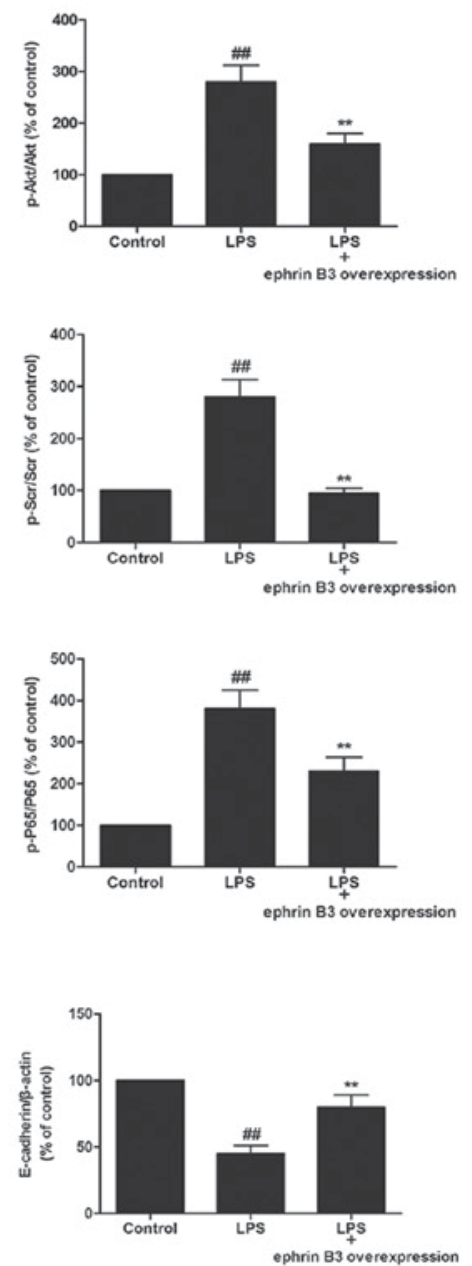

$95 \mathrm{kDa}$
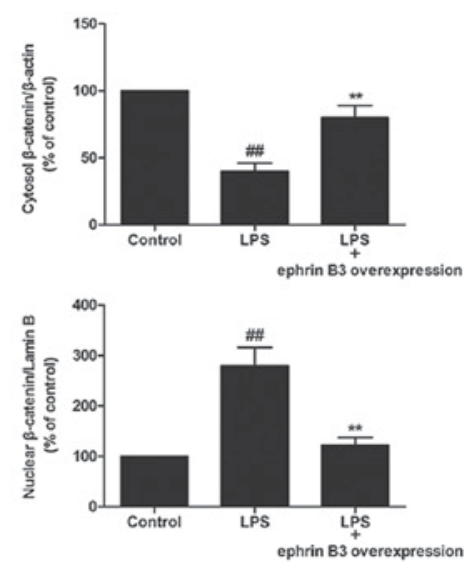

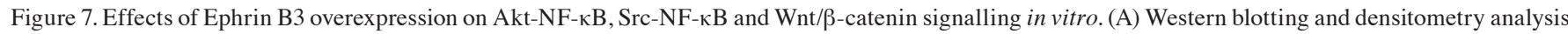
of the total expression and phosphorylation of Akt, Src and NF-kB p65. (B) Western blotting and densitometry analysis of the expression of E-cadherin and nuclear translocation of $\beta$-catenin. The results are expressed as the mean \pm standard deviation $(n=3) .{ }^{\# \#} \mathrm{P}<0.01$ vs. control; ${ }^{* *} \mathrm{P}<0.01$ vs. LPS treatment. Eph,

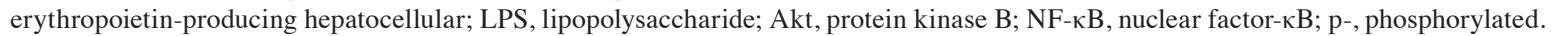

$\beta$-catenin level in the cytoplasm by recruiting Axin protein, but in the meantime Wnt signalling could also suppress the EphB-ephrinB pathway by inhibiting the transcription of ephrinB ligands (30). Therefore, we hypothesised that the maladjustment of negative feedback loops between EphrinB-dependent 'reverse' signalling and the Wnt/ $\beta$-catenin pathway might act as a crucial factor which influenced the excessive activation of the EphA2-Ephrin A1 pathway and led to LPS-induced persistent inflammation and injury. Furthermore, we speculated that there might be more interacting links between the Eph-ephrin 'forward' and 'reverse' pathways, and these bidirectional signalling networks often meditated opposing events. This may explain why LPS stimulation in a cellular context can trigger dramatically different outcomes of Eph/ephrin response.

Previous studies have proved that Eph/ephrin signaling directly activated Akt, NF- $\kappa \mathrm{B}$ and $\mathrm{Wnt} / \beta$-catenin signalling pathways (18). Crosstalk between Wnt/ $\beta$-Catenin and NF- $\kappa B$ Signaling has been reported (25). In this study, we found 
that inhibition of EphA2 or Ephrin B3 overexpression could partially alleviate LPS-triggered activation of Akt-NF- $\mathrm{kB}$, Src-NF- $\kappa B$ and Wnt/ $\beta$-catenin signalling pathways. However, the precise mechanism underlying Eph/ephrin signaling regulates Akt, NF- $\mathrm{kB}$ and $\mathrm{Wnt} / \mathrm{\beta}$-catenin signalling pathways and the crosstalk needed to be intensively investigated in future studies.

In conclusion, we demonstrated that EphA2 signalling contributes to inflammation in LPS-induced injury and that it may regulate several signalling pathways, including the Akt-NF- $\kappa \mathrm{B}$, src-NF- $\mathrm{BB}$ and $\mathrm{Wnt} / \beta$-catenin pathways. Nevertheless, EphA2 mAb treatment partially attenuated LPS-induced activation of EphA2-Ephrin A1 signalling. In addition, with regard to bidirectional signalling of the Eph-ephrins complex, we suspect that binding of EphB1 to Ephrin B3 mediated the counter-directed effect, which reduced activation of the EphA2-Ephrin A1 pathway. In the case of Ephrin B3 overexpression, two mechanisms may counteract EphA2-dependent signalling. First, Ephrin B3 overexpression decreased the expression of EphA2 and Ephrin A1. Second, the enhanced Ephrin B3-dependent'reverse' signalling led to an inactivation of the $W n t / \beta$-catenin pathway. Our results suggest that EphA2 antagonism or reinforcing Ephrin B3 binding to EphB1 might be a potentially therapeutic avenue for LPS-induced intestinal injury, and even PI-IBS. Further work regarding Eph/ephrin signalling in the PI-IBS is required to establish a therapeutic strategy with clinical potential.

\section{Acknowledgements}

Not applicable.

\section{Funding}

The present study was supported by the International Scientific and Technological Cooperation Projects of Shenzhen Collaborative Innovation Technology Plan (grant no. GJHZ20150316141713255).

\section{Availability of data and materials}

The datasets used and/or analyzed during the current study are available from the corresponding author on reasonable request.

\section{Authors' contributions}

YX, KL, HW and LZ contributed to the conception and design of the study. YX, KL, HW, LJ, SY and LZ performed the experiments to acquire the data. YX, KL, LJ, SY and LZ performed data analyses and interpreted the data.

\section{Ethics approval and consent to participate}

The present study was approved by The Institutional Animal Experiment Committee of the Second People's Hospital of Shenzhen (Guangdong, China).

\section{Patient consent for publication}

Not applicable.

\section{Competing interests}

The authors declare that they have no competing interests.

\section{References}

1. Oświęcimska J, Szymlak A, Roczniak W, Girczys-Połedniok K and Kwiecień J: New insights into the pathogenesis and treatment of irritable bowel syndrome. Adv Med Sci 62: 17-30, 2017.

2. Mearin F, Ciriza C, Mínguez M, Rey E, Mascort JJ, Peña E, Cañones P, Júdez J; en nombre de la SEPD; la semFYC, et al: Clinical practice guidelines: Irritable bowel syndrome with constipation and functional constipation in adults: Concept, diagnosis, and healthcare continuity. (Part 1 of 2). Atencion Primaria 49: 42-55, 2017 (In Spanish).

3. Sundin J, Rangel I, Repsilber D and Brummer RJ: cytokine response after stimulation with key commensal bacteria differ in Post-Infectious Irritable Bowel Syndrome (PI-IBS) patients compared to healthy controls. PLoS One 10: $\mathrm{e} 0134836,2015$.

4. Mondelaers SU, Theofanous SA, Florens MV, Perna E, Aguilera-Lizarraga J, Boeckxstaens GE and Wouters MM: Effect of genetic background and postinfectious stress on visceral sensitivity in Citrobacter rodentium-infected mice. Neurogastroenterol Motil 28: 647-658, 2016.

5. Lan C, Sun XN, Zhou XC, Yang B, Huang BL, Deng TZ, He ZT and Han XY: Preinduced intestinal HSP70 improves visceral hypersensitivity and abnormal intestinal motility in PI-IBS mouse model. Asian Pac J Trop Med 9: 302-305, 2016.

6. Polster AV, Friberg P, Neve BL, Törnblom H and Simren M: Autonomic nervous system function in patients with Irritable Bowel Syndrome (IBS): Relevance for symptoms? Gastroenterology 152 (Suppl 1): S724, 2017.

7. Menzies V, Jallo N, Kinser P, Robins JL, An K, Driscoll C, Starkweather A, Bajaj JS and Lyon DE: Shared symptoms and putative biological mechanisms in chronic liver disease: Implications for biobehavioral research. Biol Res Nurs 17: 222-229, 2015.

8. Hyland NP and Cryan JF: Microbe-host interactions: Influence of the gut microbiota on the enteric nervous system. Dev Biol 417: 182-187, 2016.

9. Rao M, Rastelli D, Dong L, Chiu S, Setlik W, Gershon MD and Corfas G: Enteric glia regulate gastrointestinal motility but are not required for maintenance of the epithelium in mice. Gastroenterology 153: 1068-1081.e7, 2017.

10. Cashman MD, Martin DK, Dhillon DK and Puli SR: Irritable bowel syndrome: A clinical review. Curr Rheumatol Rev 12: $13-26,2016$.

11. Li S, Fei G, Fang X, Yang X, Sun X, Qian J, Wood JD and Ke M: Changes in enteric neurons of small intestine in a rat model of irritable bowel syndrome with diarrhea. J Neurogastroenterol Motil 22: 310-320, 2016.

12. Park I and Lee HS: EphB/ephrinB signaling in cell adhesion and migration. Mol Cells 38: 14-19, 2015.

13. Barquilla A and Pasquale EB: Eph receptors and ephrins: Therapeutic opportunities. Annu Rev Pharmacol Toxicol 55: 465-487, 2015.

14. Chen X, Cheng ZR, Zhang SJ, Werling D and Wathes DC: Combining genome wide association studies and differential gene expression data analyses identifies candidate genes affecting mastitis caused by two different pathogens in the dairy cow. Open J Anim Sci 5: 358-393, 2015.

15. Kang M, Jeong W, Bae H, Lim W, Bazer FW and Song G: Bifunctional role of ephrin A1-Eph system in stimulating cell proliferation and protecting cells from cell death through the attenuation of ER stress and inflammatory responses in bovine mammary epithelial cells. J Cell Physiol 233: 2560-2571, 2018.

16. Han J, Xu Y, Yang D, Yu N, Bai Z and Bian L: Effect of polysaccharides from acanthopanax senticosus on intestinal mucosal barrier of escherichia coli lipopolysaccharide challenged mice. Asian-Australas J Anim Sci 29: 134-141, 2016.

17. Dun MD, Chalkley RJ, Faulkner S, Keene S, Avery-Kiejda KA, Scott RJ, Falkenby LG, Cairns MJ, Larsen MR, Bradshaw RA and Hondermarck H: PProteotranscriptomic profiling of 231-BR breast cancer cells: Identification of potential biomarkers and therapeutic targets for brain metastasis. Mol Cell Proteomics 14: 2316-2330, 2015 
18. Hong JY, Shin MH, Douglas IS, Chung KS, Kim EY, Jung JY, Kang YA, Kim SK, Chang J, Kim YS and Park MS: Inhibition of EphA2/EphrinA1 signal attenuates lipopolysaccharide-induced lung injury. Clin Sci (Lond) 130: 1993-2003, 2016.

19. Dunne PD, Dasgupta S, Blayney JK, McArt DG, Redmond KL, Weir JA, Bradley CA, Sasazuki T, Shirasawa S, Wang T, et al: EphA2 expression is a key driver of migration and invasion and a poor prognostic marker in colorectal cancer. Clin Cancer Res 22: 230-242, 2015

20. Whitman $\mathrm{E}$ and Barber $\mathrm{A}$ : NKG2D receptor activation of $\mathrm{NF}-\kappa \mathrm{B}$ enhances inflammatory cytokine production in murine effector CD8(+) T cells. Mol Immunol 63: 268-278, 2015.

21. Cheng CY, Huang WR, Chi PI, Chiu HC and Liu HJ: Cell entry of bovine ephemeral fever virus requires activation of Src-JNK-AP1 and PI3K-Akt-NF- $\mathrm{B}$ pathways as well as Cox-2-mediated PGE2/EP receptor signalling to enhance clathrin-mediated virus endocytosis. Cell Microbiol 17: 967-987, 2015.

22. Daniel AR, Gaviglio AL, Knutson TP, Ostrander JH, D'Assoro AB Ravindranathan P, Peng Y, Raj GV, Yee D and Lange CA: Progesterone receptor-B enhances estrogen responsiveness of breast cancer cells via scaffolding PELP1- and estrogen receptor-containing transcription complexes. Oncogene 34: 506-515, 2015.

23. Wu Y, Zhang Y, Wang L, Diao Z and Liu W: The role of autophagy in kidney inflammatory injury via the NF- $\kappa$ B route induced by LPS. Int J Med Sci 12: 655-667, 2015.

24. Huang J, He Y, Mcleod HL, Xie Y, Xiao D, Hu H, Chen P, Shen L, Zeng S, Yin X, et al: miR-302b inhibits tumorigenesis by targeting EphA2 via Wnt/ $\beta$-catenin/EMT signaling cascade in gastric cancer. BMC Cancer 17: 886, 2017.
25. Ma B and Hottiger MO: Crosstalk between Wnt/ $\beta$-Catenin and NF- $\kappa$ B Signaling Pathway during Inflammation. Front Immunol 7: 378, 2016.

26. Ma B, Fey M and Hottiger MO: WNT/ $\beta$-catenin signaling inhibits CBP-mediated RelA acetylation and expression of proinflammatory $\mathrm{NF}-\kappa \mathrm{B}$ target genes. J Cell Sci 128: 2430-2436, 2015.

27. Voutilainen M, Lindfors PH, Trela E, Lönnblad D, Shirokova V, Elo T, Rysti E, Schmidt-Ullrich R, Schneider P and Mikkola ML: Ectodysplasin/NF- $\kappa$ B promotes mammary cell Fate via Wnt//-catenin pathway. PLoS Genet 11: e1005676, 2015.

28. Huang H, Li R, Yuan J, Zhou X, Liu X, Ou S, Xu T and Chen Y. Up-regulated ephrinB3/EphB3 expression in intractable temporal lobe epilepsy patients and pilocarpine induced experimental epilepsy rat model. Brain Res 1639: 1-12, 2016.

29. Dong LD, Gao F, Wang XH, Miao Y, Wang SY, Wu Y, Li F, Wu J, Cheng XL, Sun XH, et al: GluA2 trafficking is involved in apoptosis of retinal ganglion cells induced by activation of EphB/EphrinB reverse signaling in a rat chronic ocular hypertension model. J Neurosci 35: 5409-5421, 2015

30. Eusemann TN, Willmroth F, Fiebich B, Biber K and van Calker D: Adenosine receptors differentially regulate the expression of regulators of G-protein signalling (RGS) 2, 3 and 4 in astrocyte-like cells. PLoS One 10: e0134934, 2015.

This work is licensed under a Creative Commons Attribution-NonCommercial-NoDerivatives 4.0 International (CC BY-NC-ND 4.0) License. 